\title{
Infrared spectra of nitric acid trihydrate ( $\beta$-NAT): A comparison of available optical constants and implication for the detection of polar stratospheric clouds (PSCs)
}

\author{
I. K. Ortega, ${ }^{1,2}$ B. Maté, ${ }^{1}$ M. A. Moreno, ${ }^{1}$ V. J. Herrero, ${ }^{1}$ and R. Escribano ${ }^{1}$ \\ Received 22 May 2006; revised 18 August 2006; accepted 30 August 2006; published 12 October 2006.
}

[1] The $\beta$ phase of nitric acid trihydrate $(\beta$-NAT) is assumed to be one of the essential components of polar stratospheric clouds (PSC) and may play an important role in ozone chemistry. Infrared spectra of vapour deposited films of $\beta$-NAT, have been measured in transmission and in reflection-absorption (RAIR). The spectra have been simulated with the different sets of optical constants available in the literature. The films are found to be optically isotropic. The refractive indices of Toon et al. (1994) give the best global fit to the data, but have low resolution and tend to smooth sharp features. The higher resolution indices of Höpfner et al. (2006) lead to worse overall spectral simulations, but can account for the comparatively narrow feature at $820 \mathrm{~cm}^{-1}$ recently identified in field measurements of $\beta$-NAT particles. The best compromise is obtained with the "coa" indices of Höpfner et al. Citation: Ortega, I. K., B. Maté, M. A. Moreno, V. J. Herrero, and R. Escribano (2006), Infrared spectra of nitric acid trihydrate ( $\beta$-NAT): A comparison of available optical constants and implication for the detection of polar stratospheric clouds (PSCs), Geophys. Res. Lett., 33, L19816, doi:10.1029/ 2006GL026988.

\section{Introduction}

[2] Nitric acid trihydrate (NAT), an essential constituent of polar stratospheric clouds (PSCs), is believed to play a relevant role in the destruction of ozone, both by providing a site for heterogeneous reactions that activate halogen species, and by contributing to denitrification through sedimentation of large particles [see, e.g., Solomon, 1999; Fahey et al., 2001; Tolbert and Toon, 2001; Voigt et al., 2005, and references therein]. The presence of nitric acid hydrates in PSCs was already conjectured in early work [Crutzen and Arnold, 1986; Toon et al., 1986] and the laboratory studies of Hanson and Mauersberger [1988a, $1988 \mathrm{~b}$ ] showed that NAT is the thermodynamically stable crystalline form of nitric acid under the conditions of the winter polar stratosphere. The direct identification of NAT in field measurements has proven however difficult. Only in 2000 were crystalline particles with the expected 3:1 molar ratio between water and nitric acid definitely detected with careful mass spectrometric procedures in type 1 PSCs [Voigt et al., 2000]. A more direct link to the structure of the cloud

\footnotetext{
${ }^{1}$ Instituto de Estructura de la Materia, Madrid, Spain.

${ }^{2}$ Also at Departamento de Química Física y Analítica, Universidad de Jaén, Jaén, Spain.
}

Copyright 2006 by the American Geophysical Union. 0094-8276/06/2006GL026988 particles could be obtained, at least in principle, from spectroscopic data, but these measurements are complicated by the concurrence of many absorbing species and by the need of reliable optical indices of the observed aerosols. Optical indices are also essential in the interpretation of observations of tropical thin cirrus clouds, which is a topic of present interest [see, e.g., Popp et al., 2006, and references therein].

[3] Transmission infrared (IR) laboratory spectra of NAT films were reported in the early nineties by Ritzhaupt and Devlin [1991] and by Koehler et al. [1992]. Koehler et al. also observed that the IR spectra of NAT changed markedly when the sample was heated above $\approx 185 \mathrm{~K}$ and proposed the existence of two distinct phases of NAT; they termed the metastable lower temperature phase $\alpha$-NAT, and the higher temperature phase $\beta$-NAT, which should be the one relevant for PSCs from a thermodynamic point of view. However the discrimination of different crystal phases from IR spectra alone is problematic and in many literature works the term NAT continued to be used without explicit mention of the $\alpha$ or $\beta$ phase. In recent studies, Tizek et al. [2004] and Grothe et al. [2006] have confirmed the existence of the two phases by associating two different X-Ray diffractograms to NAT samples characterized by $\alpha$ - and $\beta$-NAT IR spectra respectively.

[4] A first attempt to identify the condensed phase particles in type 1 PSCs by means of IR spectroscopy was reported by Toon and Tolbert [1995]. These authors tried to simulate available low resolution solar occultation spectra recorded over Antarctica, with the laboratory optical indices derived by Toon et al. [1994] for water ice and nitric acid hydrates and could not identify NAT particles.

[5] The advent of higher resolution data from limb sounding instruments has opened new opportunities for the characterization of the particles in PSCs. Various studies [Spang and Remedios, 2003; Spang et al., 2005; Höpfner et al., 2006] based on radiance measurements from CRISTA (CRyogenic Infrared Spectrometers and Telescopes for the Atmosphere) and MIPAS (Michelson Interferometer for Passive Atmospheric Sounding) could associate an IR absorption feature at $820 \mathrm{~cm}^{-1}$ with backscatter and depolarisation LIDAR data indicating solid type clouds (Type 1a). This feature was attributed to a nitrate band in $\beta$-NAT. Initial attempts to simulate the measurements with the $\beta$-NAT refractive indices of Toon et al. [1994] were not conclusive, but a recent study by Höpfner et al. [2006], using two new sets of optical constants derived from spectra of Biermann [1998], has led to a successful simulation of the $820 \mathrm{~cm}^{-1}$ peak in the satellite measurements. In the same study [Höpfner et al., 2006], the authors ruled out the 


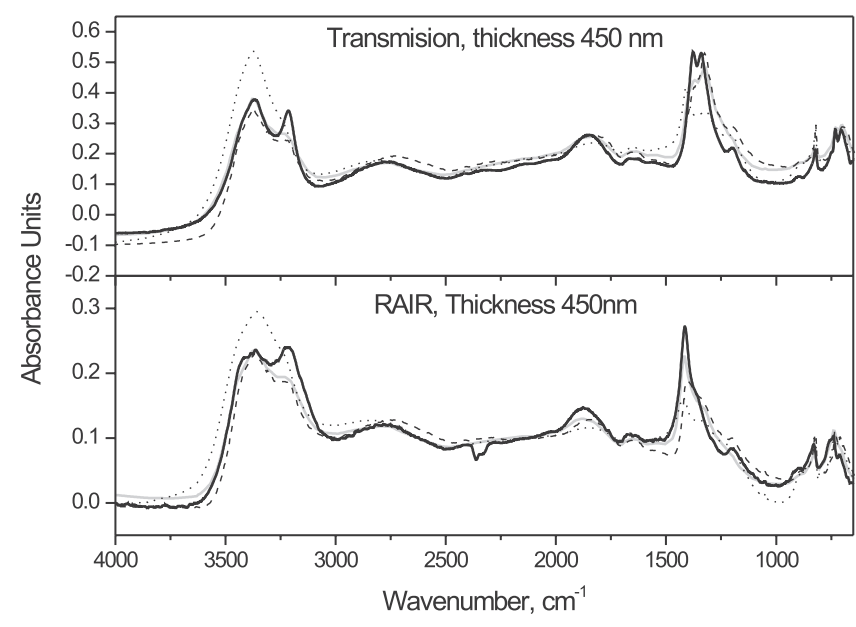

Figure 1. (top) IR transmission spectrum of a vapor deposited film of $\beta$-NAT. Black solid line: experimental data. Grey solid line: simulation of the spectrum with the optical constants from Toon et al. [1994]. Dotted line: simulation of the spectrum with the "mol" optical constants of Höpfner et al. [2006]. Dashed line: simulation of the spectra with the "coa" optical constants of Höpfner et al. [2006]. (bottom) RAIR spectrum of a vapour deposited film of $\beta$-NAT. Symbols: same as in top plot.

presence of $\alpha$-NAT and nitric acid dihydrate (NAD) in their observations.

[6] Previous investigations have shown that IR spectra of nitric acid hydrates may depend on the particular spectroscopic technique used, on the type and history of the samples investigated and also on their orientation or shape [see, e.g., Tisdale et al., 1999; Maté et al., 2004; Wagner et al., 2005, Maté et al., 2006, and references therein]. In fact, the three sets of $\beta$-NAT optical indices [Toon et al., 1994; Höpfner et al., 2006] used for the analysis of PSCs show appreciable differences, and although the particular spectral feature mentioned in the previous paragraph is better accounted for by the recent data of Höpfner et al. [2006], a global assessment of the available optical constants is timely. In this work we have examined the adequacy of the various sets of refractive indices of $\beta$-NAT for the simulation of transmission and reflection-absorption IR (RAIR) spectra, recorded both with polarized and unpolarized radiation.

\section{Experimental}

[7] The experimental set-up has been described in previous works [Carrasco et al., 2002; Maté et al., 2003, 2006]. In this section a brief description is given, with special emphasis in the details relevant for the present measurements. $\beta$-NAT films were formed by vacuum deposition on various substrates kept at $185 \mathrm{~K}$ inside a high vacuum chamber. The background pressure was in the $10^{-9} \mathrm{mbar}$ range. $\mathrm{HNO}_{3}$ and $\mathrm{H}_{2} \mathrm{O}$ were introduced in the chamber through independent inlets and the $\mathrm{HNO}_{3} / \mathrm{H}_{2} \mathrm{O}$ molar ratio in the vapour was selected to be $25 \%$ from the phase diagrams of Tizek et al. [2004]. Note that under the selected deposition conditions, the $\beta$-NAT crystalline phase is directly formed with the right $(3: 1)$ water/nitric acid proportion and no additional curing of the film is necessary. The total chamber pressure during the deposition procedure was approximately $1 \times 10^{-4}$ mbar, as measured with a capacitance manometer (Leybold, Ceravac CTR 81). The estimated film growing rates for all samples were 30$40 \mathrm{~nm} / \mathrm{min}$. The optical properties of the samples are not affected by the growing rate [see, e.g., Maté et al., 2006, Figure 1].

[8] IR spectra of the deposited samples were recorded, both in transmission and in reflection-absorption, with a Bruker IFS66 FTIR spectrometer. Ge and Si were used as deposition substrates for the transmission measurements and Al for the RAIRS experiments. The IR radiation was focused on the sample with a $\mathrm{KBr}$ lens at an incidence angle of $75^{\circ}$ for the RAIR configuration and at normal incidence for the transmission setup. The reflected or transmitted IR light was focused on a MCT detector cooled with liquid nitrogen. In the RAIR configuration a polarizer (Specac KRS-5) placed before the focusing lens, was used to select the polarization of the incident radiation. The corresponding s- or p-polarized spectra were recorded with the electric field vector perpendicular or parallel to the incidence plane, respectively. Each spectrum was obtained from the addition of 512 scans. The experiments were usually carried out with a nominal resolution of $8 \mathrm{~cm}^{-1}$ $\left(0.12 \mathrm{~cm}\right.$ mirror displacement) and, in some cases, $1 \mathrm{~cm}^{-1}$ resolution. (0.91 cm mirror displacement) A three term Blackman-Harris apodization function was used in all cases.

[9] Simulations of the spectra were performed by means of classical Fresnel models using optical constants from the literature. The simulation procedures are described in detail by Maté et al. [2003] and Fernández-Torre et al. [2005] for the RAIRS and transmission measurements respectively. The approximate widths of the different films were estimated, with an uncertainty of $\approx \pm 25 \mathrm{~nm}$, by comparison of the spectra with the best possible global simulations. In the RAIR measurements an independent estimate with a larger uncertainty $( \pm 50 \mathrm{~nm})$ was obtained from interference measurements with a $\mathrm{He}-\mathrm{Ne}$ laser [Maté et al., 2003] and both values were seen to be coincident The width given for the transmission experiments corresponds to the thickness of the film formed at each side of the substrate (i.e. one half of the total absorbing length).

\section{Results and Discussion}

[10] Figure 1 shows a transmission and a RAIR spectrum of $\beta$-NAT films of comparable thickness, together with their respective simulations with optical constants from the literature. The same spectral features can be easily recognized in both spectra and can be assigned from previous works, including recent theoretical calculations [Koehler et al., 1992; Tso and Leu, 1996; Fernández et al., 2003; Martin-Llorente et al., 2006]. The band with two maxima at frequencies beyond $3000 \mathrm{~cm}^{-1}$ is due to $\mathrm{OH}$ stretching vibrations of the water molecules and the continuum between 3000 and $1500 \mathrm{~cm}^{-1}$, with two broad maxima at 2750 and $1850 \mathrm{~cm}^{-1}$, is caused by $\mathrm{OH}$ stretching vibrations of the hydrated oxonium ion [Fernández et al., 2003; Martín-Llorente et al., 2006]. Bending vibrations of $\mathrm{H}_{3} \mathrm{O}^{+}$and $\mathrm{H}_{2} \mathrm{O}$ are found between 1750 and $1500 \mathrm{~cm}^{-1}$. The large peak at about $1400 \mathrm{~cm}^{-1}$ corresponds mostly to 
the $\nu_{3}$ antisymmetric stretching vibration of the $\mathrm{NO}_{3}^{-}$ion; the small shoulder at $\approx 1200 \mathrm{~cm}^{-1}$ is associated to the $\nu_{2}$ symmetric bending of $\mathrm{H}_{3} \mathrm{O}^{+}$, and the narrow small peak at $820 \mathrm{~cm}^{-1}$ is due to the $\nu_{2}$ symmetric bending of $\mathrm{NO}_{3}^{-}$. At lower frequencies lattice modes, sometimes mixed with molecular motions, become gradually dominant.

[11] The simulation of the transmission spectra (Figure 1, top plot) with the optical constants from the literature leads to a general good agreement, with some mismatches. All the experimental features commented on above are reproduced with the three sets of optical indices employed. The simulation with the "mol" constants of Höpfner et al. [2006] (dotted line) leads to a too high absorption in the region between 3000 and $3500 \mathrm{~cm}^{-1}$ and to a comparatively small signal for the $\nu_{3}$ stretching of $\mathrm{NO}_{3}^{-}$at $1380 \mathrm{~cm}^{-1}$. The agreement is better for the rest of the spectral range. In contrast, the simulation with the "coa" indices of Höpfner et al. [2006] (dashed line) reproduces well the height of the two main peaks, but overestimates the absorption in other parts of the spectrum. The best overall simulation is obtained with the optical constants of Toon et al. [1994] (grey trace), that lead to an excellent agreement with the measurements between 3500 and $1500 \mathrm{~cm}^{-1}$. Below this point the accordance is somewhat worse. The simulations of the RAIR spectrum (Figure 1, bottom plot) lead to an even better agreement with experiment, and only the "mol" indices present an appreciable overestimation of the peak at $3300 \mathrm{~cm}^{-1}$. The experimental "dip" at about $2300 \mathrm{~cm}^{-1}$ is due to some $\mathrm{CO}_{2}$ contamination in the IR beam line. Although both the "coa" indices and those of Toon et al. [1994] give a shoulder at $3200 \mathrm{~cm}^{-1}$, the double peak recorded in the present $\beta$-NAT measurements is not reproduced accurately in any of the simulations.

[12] All three sets of optical constants used for the reproduction of the measurements, were obtained in the original works by applying an iterative Kramers-Kronig technique to $\beta$-NAT spectra taken from different samples. The indices of Toon et al. [1994] were derived from transmission spectra of vapor deposited samples generated by a procedure analogous to that used in the present work, under very similar deposition conditions. The reported resolution of the measurements was also $8 \mathrm{~cm}^{-1}$ and the spectra were recorded at $167 \mathrm{~K}$. The "mol" indices of Höpfner et al. [2006] were obtained from normal incidence transmission spectra of a polycrystalline sample generated by cooling a $3: 1 \mathrm{H}_{2} \mathrm{O}: \mathrm{HNO}_{3}$ solution in a low temperature cell. The "coa" indices [Höpfner et al., 2006] were obtained from low angle RAIR spectra of films formed by codepositing $\mathrm{HNO}_{3}$ and water from the vapour phase and then evaporating the excess water by heating the sample to a temperature higher than the frost point of ice, but still within the stability region of NAT. The spectra used for the derivation of the "mol" and "coa" optical constants were measured at about $210 \mathrm{~K}$, with a resolution of $2 \mathrm{~cm}^{-1}$ (mirror displacement $0.5 \mathrm{~cm}$ ) [Biermann, 1998]. In spite of the different generation procedures and conditions of measurement, the three sets of indices available, even the "mol" ones, can give a reasonable global account of the measurements, but they differ appreciably in some details that can be relevant for the interpretation of field measurements. The difference between the spectra from the low temperature cell [Biermann, 1998] and those from all the vapor deposited samples [Koehler et al., 1992; Tso and Leu, 1996; Biermann, 1998] is also worth noting.

[13] In previous studies on $\alpha$-NAT and $\alpha$-NAD films [Maté et al., 2004, 2006], some features of the RAIR spectra were found to be absent in transmission measurements and the RAIR data could not be well simulated with the optical indices derived from normal incidence transmission measurements. An analysis of these results led to the conclusion that the $\alpha$-NAT and $\alpha$-NAD crystals grew on the substrate with a preferential orientation leading to optically anisotropic samples. Normal incidence transmission spectra of these films, from which the refractive indices were derived, are thus biased and contain only information about a preferential optical direction. As a consequence, a scrutiny of the films from other directions (e. g. grazing angle RAIRS) cannot be accounted for with these indices. In the present experiments, the coincidence in the spectral features of the transmission and reflection-absorption spectra (see Figure 1), and the fact that the optical indices derived from measurements at normal incidence can reproduce well the grazing incidence RAIRS data, strongly suggest that the films are formed by polycrystals oriented at random which give rise to optically isotropic samples.

[14] In order to probe orientation effects more in depth, we have also recorded RAIR spectra with polarized radiation. The results are shown in Figure 2. The top plot of Figure 2 corresponds to a thin $(250 \mathrm{~nm})$ film. The spectrum measured with p-polarized radiation (above) shows essentially the same bands as the non-polarized spectra of Figure 1. In contrast, the spectrum taken with s-polarized light (below) shows only a very weak absorption between 3000 and $3500 \mathrm{~cm}^{-1}$ due to the metal surface selection rule (MSSR) as discussed elsewhere [Maté et al., 2003]. The broad weak feature between 2000 and $1200 \mathrm{~cm}^{-1}$ is due to some contamination by water vapour in the line of the IR beam. The bottom plot of Figure 1 shows p- and s-polarized spectra corresponding to a film of $650 \mathrm{~nm}$. With increasing thickness, the MSSR is gradually relaxed and an appreciable absorption band appears also for the s polarization at the high frequency end. Note that the various sets of optical constants can reproduce reasonably well the different spectral shapes obtained in the experiments with polarized light. The worst agreement is again obtained with the "mol" indices that tend to give somewhat higher and broader bands between 3000 and $3500 \mathrm{~cm}^{-1}$ than those measured in the experiments. The results of Figure 2 corroborate the previous conclusion and indicate that the literature indices, especially those of Toon et al. [1994] and those from the "coa" set of Höpfner et al. [2006] should be adequate for the simulation of IR spectra of isotropic samples of $\beta$-NAT, including the likely particles in PSCs, irrespective of their geometry.

[15] As indicated above, the spectral peak at $820 \mathrm{~cm}^{-1}$ has been used for the identification of PSCs in field measurements. We have carried out further laboratory measurements to analyze in more detail the performance of the various optical indices for the simulation of this peak. The high resolution $\left(0.025 \mathrm{~cm}^{-1}\right.$ unapodized) of the MIPAS instrument on ENVISAT allowed the observation of absorption lines from many small gas-phase molecules. It should be noted at this point, that in the article of Höpfner et al. [2006] the spectral resolution is given as $1 / 2 \mathrm{~L}$, with $\mathrm{L}$ the 

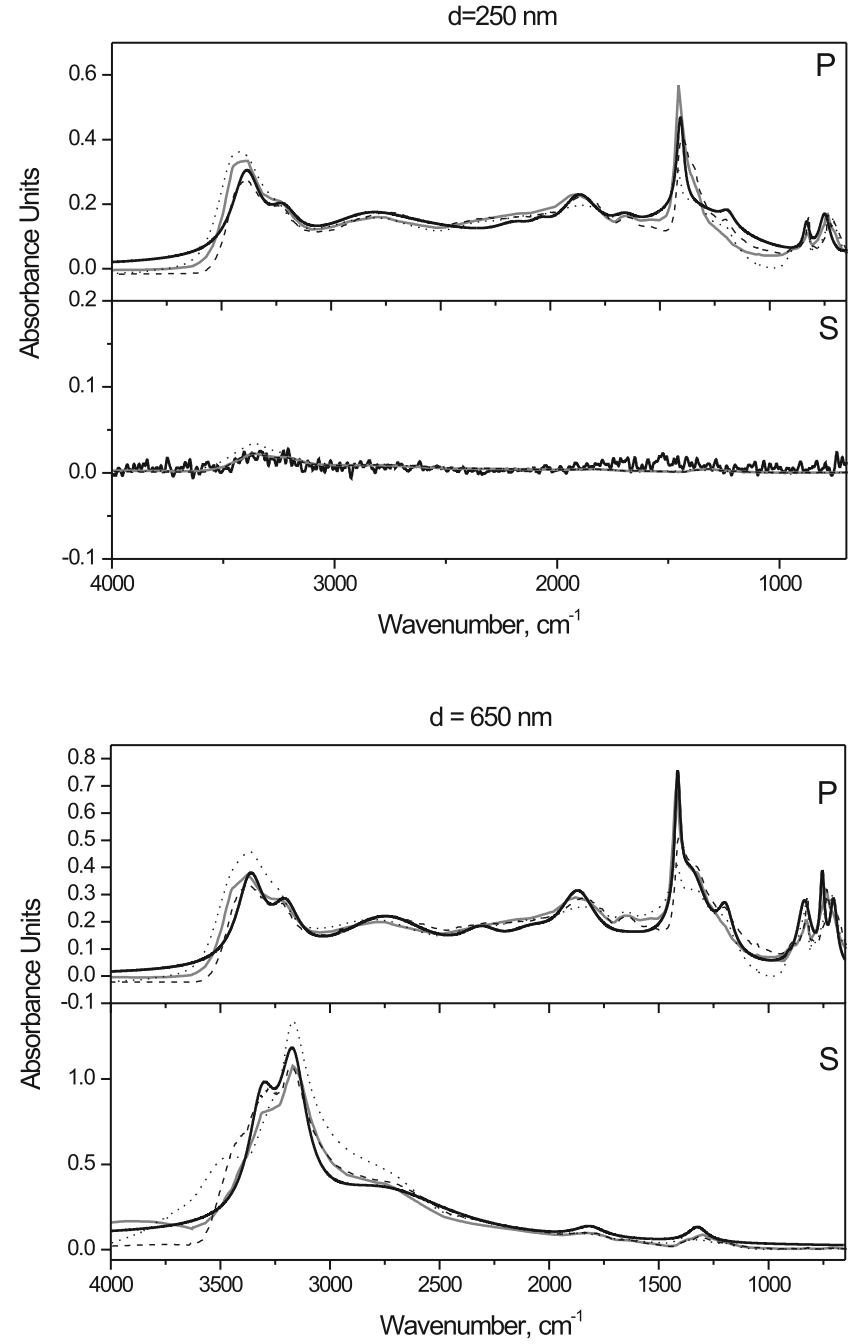

Figure 2. (top) RAIR spectrum of a thin $(250 \mathrm{~nm})$ film of $\beta$-NAT recorded with p-polarized and with s-polarized radiation. (bottom) Same as top plot but for a thicker $(650 \mathrm{~nm}) \beta$-NAT film. The different lines are defined in the caption of Figure 1.

maximum optical path. The nominal apparatus resolution used in this work (see section 2) and by Biermann [1998] is evaluated as $1 / \mathrm{L}$, yielding estimated resolution values about one half of those given by Höpfner et al. [2006] for the same mirror displacement. For a better appreciation of the aerosol features spectra of type 1a PSCs degraded to a resolution of $1 \mathrm{~cm}^{-1}$ were presented by Höpfner et al. [2006, Figure 4], where a neat peak, centered at $820 \mathrm{~cm}^{-1}$ and with an approximate full width at half maximum (FWHM) of $15 \mathrm{~cm}^{-1}$, stands out from the radiance signal. We have recorded transmission laboratory spectra of $\beta$-NAT, shown in Figure 3 for the region between 1500 and $650 \mathrm{~cm}^{-1}$, together with their corresponding simulations. The discrepancies between the different spectra become now more obvious. The top plot of Figure 3 corresponds to an $8 \mathrm{~cm}^{-1}$ nominal resolution measurement. The $820 \mathrm{~cm}^{-1}$ peak simulated with the indices of Toon et al. [1994] (grey trace) has a broader width than the experimental contour or than the simulations with the other indices, and in fact all spectral features are appreciably broadened in the simulation with these indices of Toon et al. [1994]. This result, already noted by Höpfner et al. [2006], is surprising since the optical constants of Toon et al. [1994] were derived from measurements taken also with a reported resolution of $8 \mathrm{~cm}^{-1}$. In their work, the authors do not give any details about the way the resolution is estimated and the reason for the observed discrepancy remains unclear. In contrast, the two sets of refractive indices of Höpfner et al. [2006], consist of data derived from spectra recorded with a resolution of $2 \mathrm{~cm}^{-1}$ [Biermann, 1998] $\left(1 \mathrm{~cm}^{-1}\right.$ in the criterion of Höpfner et al.) and the $820 \mathrm{~cm}^{-1}$ peak simulated with these indices is sharper than that measured with a $8 \mathrm{~cm}^{-1}$ resolution. In the bottom plot of Figure 3, the same theoretical simulations are compared to an experimental spectrum recorded with an apodized resolution of $1 \mathrm{~cm}^{-1}$. In this case the simulations with the indices of Höpfner et al. [2006] are in very good agreement with the experimental peak. However, the agreement is not always so good in other regions of the spectra. In particular, the "mol" indices are at variance with the rest of the spectra in the 1200$1400 \mathrm{~cm}^{-1}$ range (around the $\nu_{3}$ peak of $\mathrm{NO}_{3}^{-}$), which is also of interest for the results of MIPAS. The "coa" set of indices is probably the best compromise for the analysis of the field data, since it has the high resolution needed to account for the distinctive narrow feature at $820 \mathrm{~cm}^{-1}$ and gives also a reasonably good global fit to the whole mid IR spectrum of $\beta$-NAT.

\section{Conclusions}

[16] IR refractive indices from the literature can simulate reasonably well the transmission and RAIR spectra measured in this work for thin films of $\beta$-NAT deposited from the vapor phase on a substrate at $185 \mathrm{~K}$. In contrast to previous results on $\alpha$-NAT and $\alpha$-NAD, the films of $\beta$-NAT are found to be optically isotropic not showing a preferential growth orientation with respect to the substrate. This result indicates that the available optical constants can in principle be used for the simulation of any isotropic sample of $\beta$-NAT

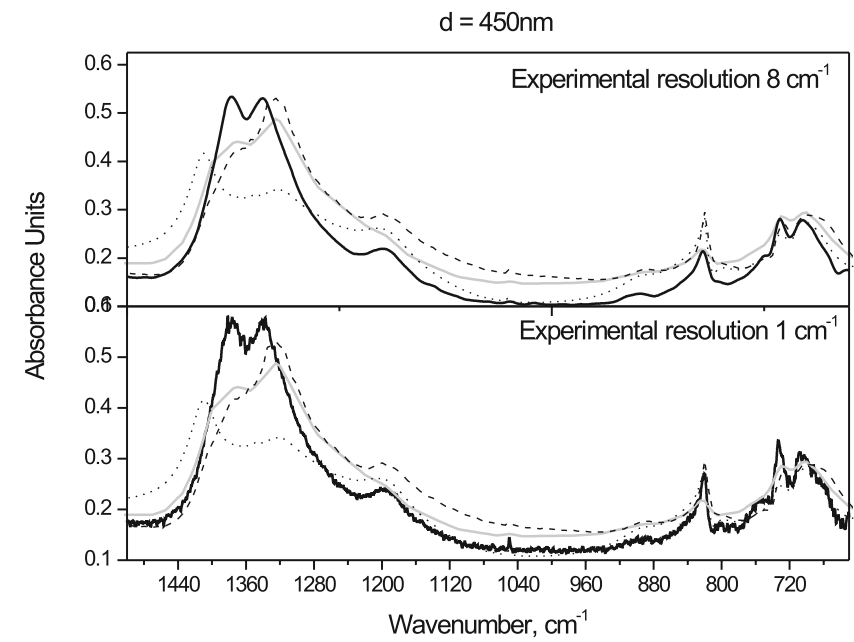

Figure 3. Transmission spectra of $\beta$-NAT in the 650 $1500 \mathrm{~cm}^{-1}$ frequency range. (top) Experimental resolution $8 \mathrm{~cm}^{-1}$. (bottom) Experimental resolution $1 \mathrm{~cm}^{-1}$. The lines of the various spectra are defined in Figure 1. 
including the likely particles in PSCs. The refractive indices of Toon et al. [1994] provide the best global simulation of the present spectra, but the data are too widely spaced in frequency and tend to smooth sharp features. In fact the narrow spectral peak at $820 \mathrm{~cm}^{-1}$, used for the identification of $\beta$-NAT in PSCs, is better accounted for by the higher resolution "coa" and "mol" indices of Höpfner et al. [2006]. Among these two sets, the "coa" indices are preferable since they provide a better simulation of the whole spectrum.

[17] Acknowledgments. We are indebted to M. Höpfner for sending us numerical lists of the "coa" and "mol" refractive indices of $\beta$-NAT. We are also indebted to J. M. Castillo and J. Rodríguez for continuing technical support, to B. Martín-Llorente for her careful reading of the manuscript and to J. L. Doménech for helpful discussions. This work has been funded by Project FIS2004-00456 of the Spanish Ministry of Education and has been performed within the framework of the "Unidad Asociada" between IEM (CSIC Madrid) and the Department of Analytical and Physical Chemistry of the University of Jaén (Spain). Finally we would also like to thank the very pertinent comments of the anonymous referees.

\section{References}

Biermann, U. M. (1998), Gefrier-und FTIR-Experimente zur Nukleation und Lebensdauer stratosphärischer Wolken, Ph.D. thesis, Univ. of Bielefeld, Bielefeld, Germany.

Carrasco, E., J. M. Castillo, R. Escribano, V. J. Herrero, M. A. Moreno, and J. Rodríguez (2002), A cryostat for low-temperature spectroscopy of condensable species, Rev. Sci. Instrum., 73, 3469.

Crutzen, P. J., and F. Arnold (1986), Nitric-acid cloud formation in the cold Antarctic stratosphere: A major cause for the springtime ozone hole, Nature, 324, 651.

Fahey, D. W., et al. (2001), The detection of large $\mathrm{HNO}_{3}$ containing particles in the winter arctic stratosphere, Science, 291, 1026.

Fernández, D., V. Botella, V. J. Herrero, and R. Escribano (2003), A theoretical study of the structure and spectra of nitric acid hydrates crystals, J. Phys. Chem. B, 107, 10,608.

Fernández-Torre, D., R. Escribano, V. J. Herrero, B. Maté, M. A. Moreno, and I. K. Ortega (2005), Theoretical calculations of refractive indices and optical effects in spectra of nitric acid and nitric acid monohydrate crystals, J. Phys. Chem. B., 109, 18,010.

Grothe, H., H. Tizek, D. Waller, and D. J. Stokes (2006), The crystallization kinetics and morphology of nitric acid trihydrate, Phys. Chem. Chem. Phys., 8, 2232.

Hanson, D., and K. Mauersberger (1988a), Vapor pressures of $\mathrm{HNO}_{3} / \mathrm{H}_{2} \mathrm{O}$ solutions at low temperatures, J. Phys. Chem., 92, 6167.

Hanson, D., and K. Mauersberger (1988b), Laboratory studies of the nitric acid trihydrate: Implications for the south polar stratosphere, Geophys. Res. Lett., 15, 855 .

Höpfner, M., et al. (2006), Spectroscopic evidence for NAT, STS, and ice in MIPAS infrared limb emission measurements of polar stratospheric clouds, Atmos. Chem. Phys., 6, 1201.

Koehler, B. G., A. M. Middlebrook, and M. A. Tolbert (1992), Characterization of model polar stratospheric cloud films using Fourier-transform infrared-spectroscopy and temperature programmed desorption, J. Geophys. Res., 97, 8065 .
Martin-Llorente, B., D. Fernández-Torre, V. J. Herrero, I. K. Ortega, R. Escribano, and B. Maté (2006), Vibrational spectra of crystalline hydrates of atmospheric relevance: Bands of hydrated protons, Chem. Phys. Lett., 427, 300-304.

Maté, B., A. Medialdea, M. A. Moreno, R. Escribano, and V. J. Herrero (2003), Experimental studies of amorphous and polycristalline ice films using FT-RAIRS, J. Phys. Chem. B, 107, 11,098.

Maté, B., I. K. Ortega, M. A. Moreno, R. Escribano, and V. J. Herrero (2004), Investigation of orientation effects in films of nitric-acid trihydrate, Phys. Chem. Chem. Phys., 6, 4047.

Maté, B., I. K. Ortega, M. A. Moreno, V. J. Herrero, and R. Escribano (2006), Orientation effects on nitric acid dihydrate films, J. Phys. Chem. $B, 110,7396$.

Popp, P. J., et al. (2006), The observation of nitric acid-containing particles in the tropical lower stratosphere, Atmos. Chem. Phys., 6, 601.

Ritzhaupt, G., and J. P. Devlin (1991), Infrared spectra of nitric and hydrochloric hydrate thin films, J. Phys. Chem., 95, 90.

Solomon, S. (1999), Stratospheric ozone depletion: A review of concepts and history, Rev. Geophys., 37, 275.

Spang, R., and J. J. Remedios (2003), Observations of a distinctive infrared spectral feature in the atmospheric spectra of polar stratospheric clouds measured by the CRISTA instrument, Geophys. Res. Lett., 30(16), 1875, doi:10.1029/2003GL017231.

Spang, R., J. J. Remedios, L. J. Kramer, L. R. Poole, M. D. Fromm, M. Müller, G. Baumgarten, and P. Konopka (2005), Polar stratospheric cloud observations by MIPAS on ENVISAT: Detection method, validation and analysis of the northern hemisphere winter 2002/2003, Atmos. Chem. Phys., 5, 679.

Tisdale, R. T., A. J. Prenni, L. T. Iraci, M. A. Tolbert, and O. B. Toon (1999), Variation of the infrared spectra of nitric acid hydrates with formation conditions: Impact on PSC identification, Geophys. Res. Lett., 26, 707.

Tizek, H., E. Knözinger, and H. Grothe 2004), Formation and phase distribution of nitric acid hydrates in the mole fraction range $\mathrm{x}\left(\mathrm{HNO}_{3}\right)<$ 0.25: A combined XRD and IR study, Phys. Chem. Chem. Phys., 6, 972.

Tolbert, M. A., and O. B. Toon (2001), Solving the PSC mystery, Science, $292,61$.

Toon, O. B., and M. A. Tolbert (1995), Spectroscopic evidence against nitric-acid trihydrate in polar stratospheric clouds, Nature, 375, 218.

Toon, O. B., P. Hamill, R. P. Turco, and J. Pinto (1986), Condensation of $\mathrm{HNO}_{3}$ and $\mathrm{HCL}$ in the winter polar stratospheres, Geophys. Res. Lett., 13, 1284.

Toon, O. B., M. A. Tolbert, B. G. Koehler, A. M. Middlebrook, and J. Jordan (1994), Infrared optical constants of $\mathrm{H}_{2} \mathrm{O}$ ice, amorphous nitric-acid solutions, and nitric acid hydrates, J. Geophys. Res., 99, 25,631.

Tso, T.-L., and M.-T. Leu (1996), Quantitative analysis of the infrared absorptivities of nitric acid ices aerosol existing in polar stratospheric clouds, Anal. Sci., 12, 615.

Voigt, C., et al. (2000), Nitric acid dihydrate (NAT) in polar stratospheric clouds, Science, 290, 1756

Voigt, C., et al. (2005), Nitric acid trihydrate (NAT) formation at low supersaturation in polar stratospheric clouds (PSCs), Atmos. Chem. Phys., 5, 1371.

Wagner, R., O. Müller, H. Saathoff, O. Stelzer, and U. Schurath (2005), Infrared spectrum of nitric acid dihydrate: Influence of particle shape, J. Phys. Chem. A, 109, 2572.

R. Escribano, V. J. Herrero, B. Maté, M. A. Moreno, and I. K. Ortega, Instituto de Estructura de la Materia, Serrano 123, E-28006 Madrid, Spain. (vherrero@iem.cfmac.csic.es) 